\title{
The Study of "Implicit Learning" in Sports Skills Learning Based on Cognitive Psychology
}

\author{
Xiao-ling WANG, Jun LIU* and Su-yan ZHANG
}

Dalian University of Technology, Dalian, PRC

${ }^{*}$ Corresponding author

Keywords: Implicit learning, Cognitive psychology, Learning disabilities, Influencing factors.

\begin{abstract}
To explore the role of "implicit learning" in sports skill learning and internal connection between it and task accepting, compressive force, follow-up hard work and others' influence, we studied the phenomenon of "implicit learning" in the study of dancesport skill. Through questionnaires, we found that students who have learning disabilities for sports have improved their ability to master skills after exerting pressure, repeating exercises, and being helped by common learners. The Conclusion is that there are implicit learning in sports dance, and positive psychological guide can improve learning results.
\end{abstract}

\section{基于认知心理学对体育类技能学习中“内隐学习” 的探究 \\ 王晓玲, 刘君 ${ }^{*}$, 张素艳 \\ 大连理工大学, 大连, 中国 \\ “通讯作者}

关键词: 内隐学习; 认知心理学; 学习障碍; 技能掌握

摘要：为探索“内隐学习”在体育技能学习中起到的作用, 以及“内隐学习”与接受任务、所受 压力、后续努力及所受他人的影响之间的内在联系, 基于认知心理学中“内隐学习”这一概念, 研究体育舞蹈的技能学习中的“内隐学习” 现象。通过问调查, 分析出对于体育技能有学习障 碍的学生在施压, 重复练习以及受到共同学习者的帮助引导后技能掌握情况有所提高。得出 结论：体育舞蹈中存在内隐学习现象, 通过积极的心理引导可以改善学习情况。

\section{1. 引言}

内隐学习是认知心理学的一个重要概念, 它所指的是人类无意识地获得复杂知识的过程。 在自己未经刻意学习在不知不觉中获得某种知识, 习得了某种潜在的规则。虽然很难描述, 但是这种学习的效果可以通过某种测试表现出来, 是在意识层面无法觉知的一种规则。最早 “内隐学习”现象的研究开始于人工语法、序列规则以及复杂系统控制范式。被测试者在这些 实验当中, 似乎能够学习到一些规则, 但是要他们说出来, 又无法清晰的表示出来, 然而在 间接测试中却能够以高出随机的概率表现出学习到的规律。

近年来在高校中体育舞蹈这类动作难度较大，感情色彩比较复杂的体育技能开始普及， 高校大学生可以在一段时间内较好的配合完成一支舞, 甚至有人可以比较有感情有韵律的完 成。那么最初在语言学习以及复杂的艺术学习中被经常提到的"内隐学习"情况是否也在体育 技能性学习中存在? 如果存在, 这种"内隐学习"发挥的作用有多大, 对于不同学习状况的人又 有多大影响, 它的影响因素有哪些, 又会对体育技能性运动的学习起到哪些影响, 这种现象 的存在会给体育教学带来哪些影响, 哪些启发? 


\section{2. 研究设计与假设}

对于在校高校大学生进行随机抽样调查 204 份, 其中在进行最基础的体能训练的大学生 中抽取 111 人进行调查, 之后在学习体育舞蹈, 健美操, 跆拳道技能性的大学生中抽取 93 人 进行问调查。由不同难度的体育技能学习过程中得出是否在体育技能的学习中存在内隐学习 现象, 然后由学习者对于自己在学习体育类技能性运动的前中后三个时的描述, 及回答的相 关选择题得出在学习过程中何时“内隐学习”现象比较明显。

假设: “内隐学习”现象是一种普遍现象, 存在于所有的学习过程中, 内隐学习现象在技 能学习后发挥更大的作用, 表现也更为明显。内隐学习与平时的练习程度有很大关联。此外, 内隐学习与接受任务, 身边人的影响以及所受的压力因素也存在一定的联系。

\section{3. 研究方法}

\section{1 研究对象}

本研究选取参加体育选课的 204 名高校大学生, 进行不同难度, 不同类型的体育技能的 学习。

\section{2 测量工具}

\subsection{1 问的编制}

以认知心理学中的“内隐学习”为理论基础, 结合运动心理学相关知识, 编制关于技能学 习前情况，后“隐性学习”发挥的作用，影响因素。问以选择和简答混合的方式，问中先通过 选择对答者进行引导, 辅以简答题, 以更清晰明确的表达被调查者的状况。

\section{2 .2 问效度检验}

本实验的效度的单项与总和相关效度分析达到要求, 没有产生天花板效应或者是地板效 应, 就表面效度来说, 研究者认为问是符合要求的。

\section{3 实验设计与操作}

测试问针对 204 名学习不同体育项目的大学生展开, 其中有 111 名学生进行的是最基础 的体能训练, 其余 93 名学生分别学习体育舞蹈, 跆拳道, 健美操技能性体育运动, 将大学生 分成“前学习障碍组”和“前学习无碍组”然后对两组学生后分别分配学习任务, 共同学习技能 的同学对其产生的影响, 后续努力相对于前的努力的效率高低, 再调查学生的技能掌握情况。 从而探究技能性学习和非技能性学习之间内隐学习的存在状况以及影响因素。统计结果如图 1、图 2、图 3、图 4 及表 1 所示。

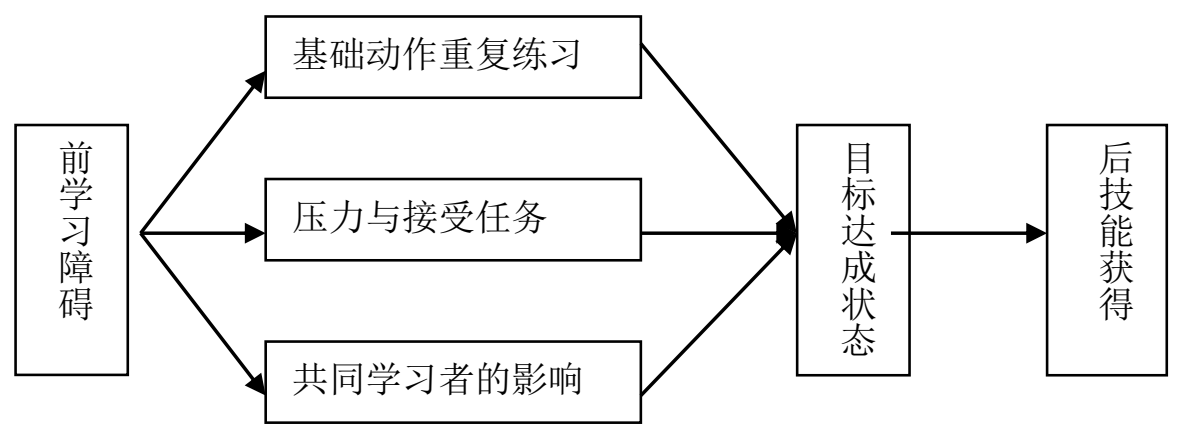

图 1 前学习障碍组的预学习过程

表 1 障碍组和无障碍组在非技能型学习 (N1) 和技能型学习 (N2) 中的对比

\begin{tabular}{lllll}
\hline & $\mathrm{N} 1$ & $\%$ & $\mathrm{~N} 2$ & $\%$ \\
\hline 前学习障碍 & 57 & 51.35 & 51 & 54.84 \\
前无障碍 & 54 & 61.65 & 42 & 45.16 \\
\hline & $\mathrm{N} 1$ & $\%$ & $\mathrm{~N} 2$ & $\%$ \\
\hline 课后增加练习 & 33 & 57.89 & 35 & 68.63 \\
\hline
\end{tabular}




\begin{tabular}{lllll}
\hline 无课后练习 & 24 & 42.11 & 16 & 31.37 \\
\hline & $\mathrm{N} 1$ & $\%$ & $\mathrm{~N} 2$ & $\%$ \\
\hline 后进步明显 & 45 & 78.95 & 40 & 78.43 \\
后变化不大 & 12 & 21.05 & 11 & 21.57 \\
\hline
\end{tabular}

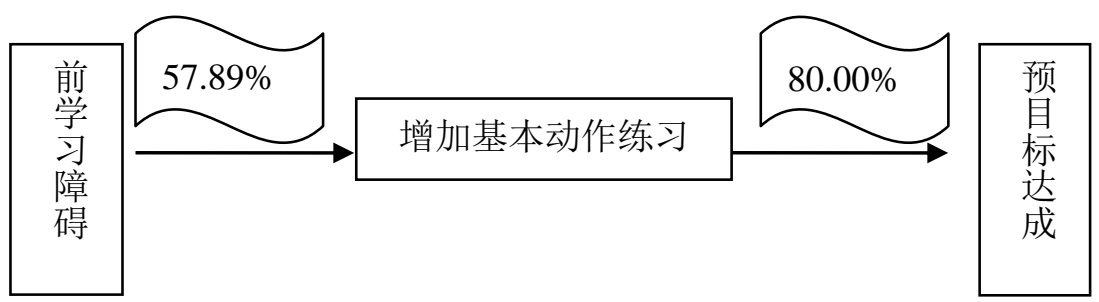

图 2 通过基本动作练习目标达成情况

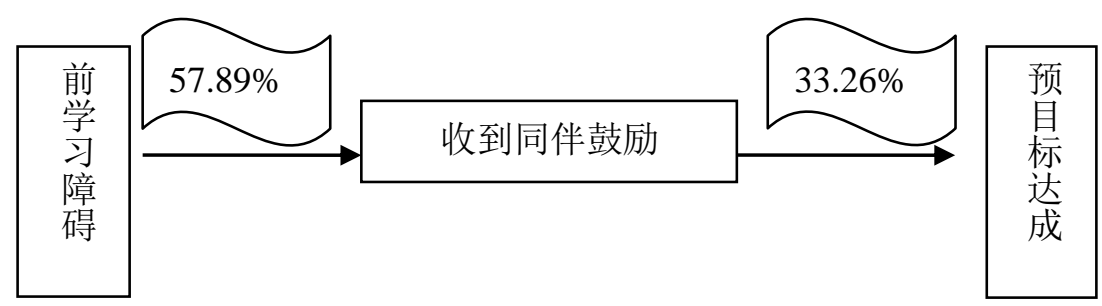

图 3 通过同伴鼓励目标达成情况

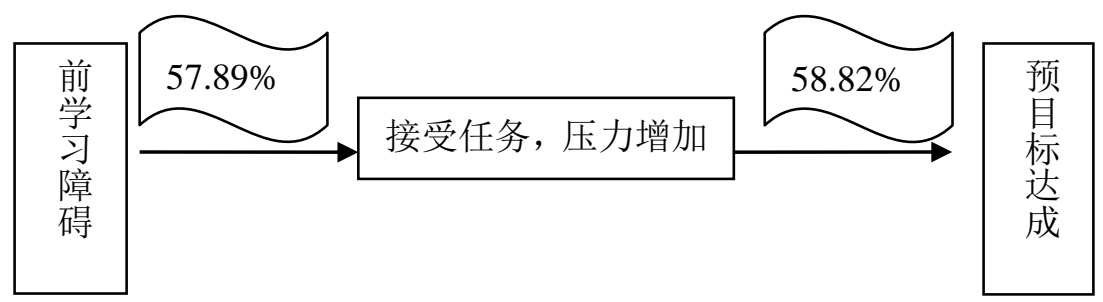

图 4 通过施加压力目标达成情况

\section{4. 研究结果}

\section{1 数据分析}

在所调查大学生中, 有大概一半左右存在前存在学习障碍, 增加基本动作练习, 收到同 伴鼓励以及接受任务, 压力增加均有助于后目标的达成, 其中增加基本动作练习在达成目标 的学生中占比为 $80 \%$, 起到主导因素作用, 其次, 接受任务, 感到压力在达成目标的学生中 占比为 $58.28 \%$, 也是主要因素之一。共同学习者在达成目标的学生中占比为 $33.26 \%$, 占比 重较小, 该外在因素对于“内隐学习”现象也起到一定作用, 但并非主要因素。

\section{2 “内隐学习” 的影响因素整合分析}
（1）反复的基础动作练习
(2) 接受任务, 压力问题
（3）共同学习者的影响 


\section{5. 讨论}

\section{1 内隐学习现象特征}

关于内隐学习以及相关的内隐认知系统的特征问题, 目前研究者之间仍有一定分歧。《内 隐学习: 理论和实践问题》中认为内隐学习最基本的特征有五点: 影响;

(1)强有力性: 外显学习和外显记忆受心理损伤的干扰, 而内隐学习和内隐记忆则不受其

(2)年龄独立性：与外显学习不同，内隐学习没有年龄和发展水平效应;

(3)低变异性: 内隐认知能力具有较小的个体间差异;

(4)独立性: 跟外显学习不同, 内隐任务成绩与标准心理测量工具测得的智力无一致性;

(5)加工的共同性：内隐学习深层的加工过程具有物种间共同性。

本次实验中我们在体育技能类运动的研究中, 通过对体育技能类与非技能类运动的比较 中也发现“内隐学习”现象与所学技能种类基本没有关系, 在技能类与非技能类的体育运动中 所存在的比较明显的“内隐学习”现象均约为 $1: 1$ 。而且“内隐学习”现象在体育类运动中也是普 遍存在的, 只是在一些人身上表现的更为明显。对于这些表现更为明显的人所填写的调查问 我们进一步分析了解到。首先, 重复练习对于技能的获得起着至关重要的作用, 同时这种基 础性的重复练习在后成果的展现起着更为突出的表现, 也就是说在“内隐学习”现象中, 累积 效应是非常重要的组成部分。其次, 共同学习者带来的影响也很重要, 比如在体育舞蹈中相 当一部分人收到舞伴的影响很大。最后, 在末接受到任务, 有更为明确的目标之后, 压力感 增加同时这种内隐学习现象表现得更为明显。

\section{2 研究可能存在的不足或有待改正之处}

本次研究中没有在生物学相关的角度进行深入的探讨与研究, 只是得到了相关性的普适 规律, 并没有研究内隐学习这种现象的存在究竟是源于什么, 内隐学习是否是与某些特殊的 神经系统的协调有关, 没有通过长时间试验研究具体哪些措施对于教学有建设性意义, 只能 给出一些方向性建议。

\section{6. 结论}

体育舞蹈学习存在内隐学习现象, 内隐学习存在积累效应, 在后的学习中体现的更加明 显。高校技能类体育运动的教学过程中可以多关注“内隐学习”现象, 在教学过程中加强基础 性动作的练习，同时更多的关注学生在学习后的表现，在适当范围内给予一些压力。注重同 学之间的合作互助关系, 此外教师在教学中所扮演的角色不仅仅是教学者, 也要更多的在心 理方面给予学生认知上更为积极的引导。

\section{致谢}

本文为“中央高校基本科研业务费资助的成果之一（DUT18RW206）。

\section{References}

[1] Smiley, Patricia A, Buttitta, Katherine V. Chung, Samuel Y. Mediation models of implicit theories and achievement goals predict planning and withdrawal after failure. MOTIVATION AND EMOTION: 40: 6: 878-894: DEC 2016

[2] Qian, Ting; Jaeger, T. Florian; Aslin, Richard N. Incremental implicit learning of bundles of statistical patterns. COGNITION: 157: 156-173: DEC 2016

[3] Zhang Wei J, Implicit Learning and Its Characteristics [K] J Journal of East China Normal University (Eductional Sciences) 
[4] Liu Yaozhong J, The Essential Features of Implicit Learning [K] J Journal of Zhanjiang Normal College (Philosophy and Social Science Edition)

[5] Liu Yaozhong J, Model of Memory System Construction Based on System Theory and Processing [K] J Journal of East China Normal University (Eductional Sciences)

[6] Vol.17, phase 1 Journal of Nanjing Sport Institute, Study on the Acquisition of Implicit Learning and Motor Skills

[7] A.S Reber (1993). Implicit Learning and Tacit Knowledge

[8] Stock, Ann-Kathrin; Steenbergen, Laura; Colzato, Lorenza. The system neurophysiological basis of non-adaptive cognitive control: Inhibition of implicit learning mediated by right prefrontal regions: HUMAN BRAIN MAPPING: 37: 12: 4511-4522: DEC 2016

[9] Branigan, Holly P.; Messenger, Katherine. Consistent and cumulative effects of syntactic experience in children's sentence production: Evidence for error-based implicit learning: COGNITION: 157: 250-256: DEC 2016

[10] A. O' Brien - Malone M. Maybery. Implicit Learning [A]. 1998.

[11] Huang Yingfeng; Huang Yushan, Implicit Learning and Grasping the Physical Movement Skills, The core collection

[12] Journal of Zhanjiang Normal College (Philosophy and Social Science Edition), 2003, Vol.17(1), pp. $22-28$

[13] A.S. Reber. Implicit learning and Tacit Knowledge: An Essay on the Cgnitive Unconscious [M]. 1993.

[14] M.J. Nissen P. Bullemer. Attentional Requirements of Learning: Evidence from Performance Measure [J]. 1987,19 .

[15] D. C. Berry Z. Dienes. Implicit Learning: Theoretical and Empirical Issues [M]. 1993. 